\title{
Nucleophile aromatische Substitution - ein neuer \\ Weg zur Synthese von Biphenylen ${ }^{[1]}$
}

Von Franz Effenberger, Klaus Nagel und Wolfgang Agster ${ }^{[*}$

Die wichtigsten Methoden zur Darstellung von Biphenylen sind die über radikalische oder metallorganische Zwischenstufen verlaufenden Ullmann- ${ }^{[2]}$ und Gomberg-Reaktionen $^{[3]}$; daneben werden Biaryle bei der Benzidinumlagerung ${ }^{[4]}$ und der oxidativen Dimerisierung ${ }^{[5]}$ sowie bei Reaktionen über Arin-Zwischenstufen ${ }^{[6]}$ gebildet.

Bei der Umsetzung von Aminobenzolen (1) mit reaktiven Halogenaromaten (2) ist uns jetzt eine polare Aryl-ArylVerknüpung durch nucleophile aromatische Substitution $\mathrm{zu}$ den Biphenylen (4) bis (6) gelungen.

Der C-C-Bindungsknüpfung vorgelagert ist in allen Fällen die Bildung von charge-transfer-(CT)-Komplexen (3), die sich bei milden Reaktionsbedingungen zum Teil in kristalliner Form isolieren lassen. Erst beim Erhitzen rea-

[*] Prof. Dr. F. Effenberger, Dipl.-Chem. K. Nagel und cand. chem. W. Agster

Institut für Organische Chemie der Universität

7 Stuttgart, Azenbergstraße 14-18 
gieren sie unter $\mathrm{HCl}$-Abspaltung zu den Biphenylen (4) bis (6) (Tabelle 1). Die Reaktivität der Komponenten (1) und (2) ist in weiten Grenzen variabel. Bei der Umsetzung von Tripyrrolidinobenzol mit Chlortrinitrobenzol (2a) kommt die Reaktionslösung zum Sieden. Durch Einführung eines Alkylrestes $\mathrm{R}^{2}$ in das Aminobenzol (I) werden die beiden ortho-ständigen Pyrrolidinoreste gegen die Aromatenebene verdrillt ${ }^{[7.8]}$; aufgrund der verringerten mesomeren Wechselwirkung sinkt die Elektronendichte und damit die Reaktivität an den freien Ringpositionen.
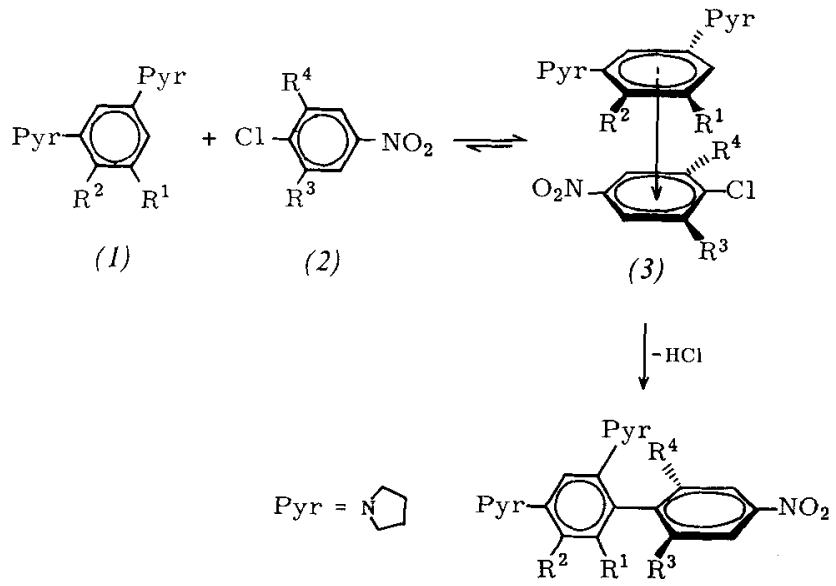

(4) $-(6)$

\begin{tabular}{lllll} 
& $\mathrm{R}^{1}$ & $\mathrm{R}^{2}$ & $\mathrm{R}^{3}$ & $\mathrm{R}^{4}$ \\
\hline$(4 a)$ & $\mathrm{Pyr}$ & $\mathrm{CH}_{3}$ & $\mathrm{NO}_{2}$ & $\mathrm{NO}_{2}$ \\
$(5 a)$ & $\mathrm{Pyr}$ & $\mathrm{H}$ & $\mathrm{NO}_{2}$ & $\mathrm{NO}_{2}$ \\
$(5 b)$ & $\mathrm{Pyr}$ & $\mathrm{H}$ & $\mathrm{CN}$ & $\mathrm{NO}_{2}$ \\
$(5 c)$ & $\mathrm{Pyr}$ & $\mathrm{H}$ & $\mathrm{COOCH}$ & $\mathrm{NO}_{2}$ \\
$(5 d)$ & $\mathrm{Pyr}$ & $\mathrm{H}$ & $\mathrm{COOH}$ & $\mathrm{NO}_{2}$ \\
$(5 e)$ & $\mathrm{Pyr}$ & $\mathrm{H}$ & $\mathrm{H}$ & $\mathrm{NO}_{2}$ \\
$(5 f)$ & $\mathrm{Pyr}$ & $\mathrm{H}$ & $\mathrm{H}$ & $\mathrm{CN}^{2}$ \\
$(6 a)$ & $\mathrm{H}$ & $\mathrm{H}$ & $\mathrm{NO}_{2}$ & $\mathrm{NO}_{2}$ \\
$(6 b)$ & $\mathrm{H}$ & $\mathrm{H}$ & $\mathrm{COOCH}_{3}$ & $\mathrm{NO}_{2}$ \\
\hline
\end{tabular}

So muß bei der Umsetzung von 1-Methyl-2,4,6-tripyrrolidinobenzol mit ( $2 a$ ) bereits erhitzt werden; mit 1- Äthyl2,4,6-tripyrrolidinobenzol bleibt die Reaktion auf der Stufe des CT-Komplexes stehen. Für die Halogenaroma-

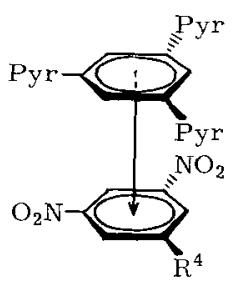

$$
\begin{aligned}
& (7 a), \mathrm{R}^{4}=\mathrm{NO}_{2} \\
& (7 e), \mathrm{R}^{4}=\mathrm{H}
\end{aligned}
$$

längstwellige Absorption von ( $7 \mathrm{a})\left(630 \mathrm{~nm}\right.$ in $\mathrm{CH}_{2} \mathrm{Cl}_{2}$ )

$$
\begin{array}{lll}
7 \times 10^{-3} & 7 \times 10^{-4} & 7 \times 10^{-5} \\
0.1 \mathrm{~cm} & 1.0 \mathrm{~cm} & 10.0 \mathrm{~cm} \\
0.31 & 0.05 & \text { ca. } 0.01
\end{array}
$$

ten liegt die Grenze der Reaktionsfähigkeit gegenüber Tripyrrolidinobenzol etwa bei der 2-Chlor-4-nitrobenzoesäure.

Die Struktur der neuen Biaryle ist durch Elementaranalyse, Molekulargewichtsbestimmung und NMR-Spektren ge- sichert; ïberraschend ist die Farbe der Verbindungen (4) bis (6), die als dunkelrote bis schwarze, glänzende Kristalle anfallen. Lage, Intensität und Struktur der farbbestimmenden Absorptionen im Sichtbaren (Tabelle 1) legen eine Deutung als charge-transfer-Banden nahe. Intermolekulare CT-Wechselwirkung ist auszuschließen, da das Lambert-Beersche Gesetz auch bei niedrigen Konzentrationen eindeutig erfïllt ist. Zur Kontrolle haben wir vergleichbare intermolekulare CT-Komplexe (7) hergestellt, die erwartungsgemäß mit sinkender Konzentration zunehmende Dissoziation zeigen.

Die weitgehend fixierte Geometrie der Biphenyle (4) bis (6) erlaubt eine genauere Untersuchung des intramolekularen Ladungsübergangs, über die in der anschließenden Mitteilung ${ }^{[9]}$ berichtet wird.

Tabelle 1. Biphenyle (4) bis (6) aus Aminobenzolen (1) und Chlorbenzolen (2)

\begin{tabular}{lllll}
\hline & $\begin{array}{l}\text { Ausb. } \\
(\%)\end{array}$ & $\begin{array}{l}\text { Fp. } \\
\left({ }^{\circ} \mathrm{C}\right)\end{array}$ & \multicolumn{2}{c}{$\begin{array}{c}\lambda_{\max }(\mathrm{nm}) ; \\
\left(\text { Molarextinktion) in } \mathrm{CH}_{2} \mathrm{Cl}_{2}\right.\end{array}$} \\
\hline$(4 a)$ & 78 & $189-190$ & $572.5 ;(3860)$ & $424.5 ;(4870)$ \\
$(5 a)$ & 79 & $174-176$ & $587 ;(6300)$ & $414 ;(3560)$ \\
$(5 b)$ & 76 & 203 & $560 ;(6140)$ & $416 ;(2180)$ \\
$(5 c)$ & 57 & 186 & $549 ;(5700)$ & sh \\
$(5 d)$ & 59 & 198 & ca. $535 ;(5 b)$ & 426.5 \\
$(5 e)$ & 85 & $209-210$ & $524.5 ;(6690)$ & $395 ;(2650)$ \\
$(5 f)$ & 64 & 212 & $484.5 ;(5680)$ & $405 ;(1440)$ \\
$(6 a)$ & 77 & 179 & $563 ;(6820)$ & $427 ;(3200)$ \\
$(6 b)$ & 84 & 148 & $523 ;(6650)$ & sh \\
\hline
\end{tabular}

Methyl-2,4-dinitro-2',4'-dipyrrolidinobiphenyl-6-carboxylat

$\mathrm{Zu} 6.48 \mathrm{~g}(0.03 \mathrm{~mol})$ 1,3-Dipyrrolidinobenzol in $15 \mathrm{ml}$ $\mathrm{CHCl}_{3}$ wird eine Lösung von $3.66 \mathrm{~g}(0.016 \mathrm{~mol})$ Methyl-2chlor-3,5-dinitro-benzoat $(2 c)$ in $15 \mathrm{ml}$ Äthanol gegeben. Die Reaktionsmischung, die sich sofort dunkelrot färbt, wird kurz aufgekocht und mit $50 \mathrm{ml}$ Äthanol versetzt. Beim Abkühlen fallen nahezu schwarze Kristalle des CT-Komplexes (3) aus $\left[\mathrm{R}^{1}=\mathrm{R}^{2}=\mathrm{H}, \mathrm{R}^{3}=\mathrm{COOCH}_{3}\right.$, $\left.\mathrm{R}^{4}=\mathrm{NO}_{2}\right]$. Ausbeute $84 \%, \mathrm{Fp}=79^{\circ} \mathrm{C}$, UV $\left[3.3 \times 10^{-3} \mathrm{M}\right.$ in $\left.\mathrm{CH}_{2} \mathrm{Cl}_{2}: \lambda_{\max }=537 \mathrm{~nm}, \mathrm{E}=0.27\right]$. (3) wird durch Erwärmen wieder in der Mutterlauge gelöst und 4 Std. unter Rückfluß erhitzt. Das Biphenyl $(6 b)$ fällt nach dem Abkühlen in dunkelroten Kristallen aus ; es kann aus Äthanol/ Petroläther umkristallisiert werden.

Eingegangen am 17. Mai 1971 [Z 443a]

[1] Für die Förderung dieser Arbeit danken wir der Deutschen Forschungsgemeinschaft und dem Fonds der Chemischen Industrie.

[2] R. G. R. Bacon u. H. A. O. Hill, Quart. Rev. 19, 95 (1965).

[3] W. E. Bachmann u. R. A. Hoffman, Org. Reactions 2, 224 (1944).

[4] H. J. Shine in B.S. Thyagarajan: Mechanisms of Molecular Migrations. Interscience, New York 1969, S. 191

[5] R. N. Adams, Accounts Chem. Res. 2, 175 (1969); dort weitere Literaturhinweise; E. C. Taylor, F. Kienzle u. A. McKillop, J. Amer Chem. Soc. 92, 6088 (1970)

[6] R.W.Hoffmann: Dehydrobenzene. Verlag Chemie, Weinheim 1967 , S. 106

[7] W. Schoeller, Dissertation, Universität Stuttgart 1969;W. D. Stohrer, Dissertation, Universität Stuttgart 1969.

[8] F. Effenberger, E. Auer u. P. Fischer, Chem. Ber, 103, 1440 (1970).

[9] E. Daltrozzo, F. Effenberger u. P. Fischer, Angew. Chem. 83, 621 (1971); Angew. Chem. internat. Edit. 10, Nr. 8 (1971). 